\title{
Paisagem sonora como conceito: tudo ou nada?
}

Thais Amorim Aragão (Universidade Federal do Rio Grande do Sul, Porto Alegre, Rio Grande do Sul, Brasil)

thaisaaragao@gmail.com

Resumo: Neste artigo, serão discutidos questionamentos que vêm emergindo nos últimos anos sobre paisagem sonora e sobre como ela vem sendo usada academicamente. O objetivo é compor um quadro crítico para uma melhor compreensão do que implica evocar essa ideia em pesquisas sobre som nos dias de hoje, cinquenta anos depois do surgimento do World Soundscape Project na Simon Fraser University. Esse quadro pode nos ajudar a articular de maneira mais adequada a ideia de paisagem sonora ou eventualmente reavaliar a necessidade de convocá-la, em favor do rigor em sua adoção como conceito e na condução de análises em que ela é tomada como parâmetro.

Palavras-chave: Paisagem sonora; Som; Epistemologias do som.

\section{Soundscape as a concept: All or nothing?}

\begin{abstract}
Recent analyses on the term soundscape and how it has been used academically will be discussed in this text. The aim is to build a conceptual framework for a better comprehension on the implications of evoking this idea on researches on sound currently, fifty years after the creation of the World Soundscape Project at Simon Fraser University. This framework may help to more adequately articulate the notion of soundscape, or eventually to even reassess the necessity of invoking it, favouring rigor in its adoption as a concept.
\end{abstract}

Keywords: Soundscape; Sound; Sonic epistemologies.

\section{Introdução}

Há cerca de cinquenta anos, entre o fim dos anos 1960 e o início da década seguinte, o compositor, educador e pesquisador canadense R. Murray Schafer estabeleceu na Simon Fraser University, Canadá, o grupo de pesquisa e educação chamado World Soundscape Project. A partir daquele momento, cursos foram oferecidos, livros foram publicados e investigações locais e intercontinentais foram realizadas, sempre mobilizando a noção emergente de paisagem sonora (tradução de soundscape para a língua portuguesa). Desde então, o termo tem inspirado inúmeros trabalhos acadêmicos pelo mundo, tornando-se uma das mais influentes ideias que hoje permeiam os estudos sobre o som. No presente artigo, levantaremos a seguinte indagação: como a paisagem sonora tem sido tratada conceitualmente?

Em busca de respostas possíveis para essa questão, serão apresentadas e ponderadas algumas análises recentes, de cunho epistemológico, desenvolvidas em torno do termo "paisagem sonora”. O intuito é compor um quadro crítico que contribua para a compreensão das implicações de evocar essa ideia em trabalhos sobre som. Múltiplos entendimentos podem ser identificados atualmente no âmbito das pesquisas acadêmicas sem que as diferenças entre eles sejam abordadas de maneira mais explícita.

Observaremos em que as miradas críticas aqui reunidas se complementam e em que elas se distanciam, sob aspectos como a consideração, ou não, de paisagem sonora como um conceito, além do entendimento sobre aquilo a que o termo se refere e as consequências de diferentes articulações para o conhecimento produzido. Adicionalmente, serão destacadas associações da problemática analisada com questões pertinentes a outros campos disciplinares, como Ciências Sociais, Planejamento Urbano e Regional, Geografia e Comunicação. 
Partiremos principalmente dos trabalhos de Tim Ingold (2011), Ari Y. Kelman (2010) e Jonathan Sterne $(2013,2015)$. No caso deste último autor, iremos um pouco mais além, analisando dois de seus textos entre si. Ambas as obras foram publicadas em um intervalo relativamente curto, de dois anos, tendo o segundo texto se constituído não apenas como uma ampliação do primeiro, como apontou o próprio Sterne (2015), mas também como uma reformulação de algumas das ideias inicialmente postas. Reconstituiremos a edição dos parágrafos, a troca de palavras, identificando acréscimos e desaparecimentos para entender como o pensamento do autor se alterou e em que direções essas mudanças apontam para concebermos a ideia de paisagem sonora.

As investigações aqui reunidas oferecem-nos mais perguntas que respostas, mas podem-nos ajudar a articular mais adequadamente a ideia sob escrutínio, desenvolvendo-a ou mesmo reavaliando a necessidade de seu uso, em favor de um maior rigor na sua adoção e na condução de análises em que a paisagem sonora é tomada como parâmetro. Tais movimentos têm o potencial de nos levar a epistemologias cientificamente mais sólidas para lidar com questões acerca do som.

\section{Um conceito que levaria ao erro}

O termo paisagem sonora começou a surgir na obra de Murray Schafer em publicações como Ear Cleaning: Notes for an Experimental Music Course (1967) e The New Soundscape: A Handbook for the Modern Music Teacher (1969). Entre os anos de 1973 e 1974, o autor foi comissionado para a produção de dez programas de rádio intitulados Soundscapes of Canada e, no ano seguinte, o World Soundscape Project visitou vilarejos europeus em cinco países para realizar um estudo sobre seus ambientes sonoros. Essa pesquisa foi publicada em 1977 como Five Village Soundscapes (KELMAN, 2010; STERNE, 2015; TRUAX, 2009).

Mas é The Tuning of the World o mais conhecido trabalho de Schafer sobre a paisagem sonora, sendo hoje um dos livros mais referenciados em estudos que envolvem de alguma forma a relação entre som e espaço ${ }^{1}$. Em inglês, foi editado novamente em 1993 como The Soundscape: Our Sonic Environment and the Tuning of the World. Por isso, eventualmente se encontram citações a ele em artigos, sobretudo em inglês, como The Soundscape, tratandose basicamente da mesma obra ${ }^{2}$.

Embora críticas tenham começado a surgir particularmente a partir de sua reedição, ainda na década de 1990, foi apenas no presente século que elas passaram a ter mais regularidade, coincidindo com o próprio estabelecimento dos chamados estudos do som. $\mathrm{O}$ debate veio a incidir sobre limitações da ideia de paisagem sonora, embaraços observados em pesquisas que nela se apoiam e o impacto desses fatores no próprio desenvolvimento do campo de estudo que tem no livro de Schafer um de seus textos fundadores.

Em 2007, o antropólogo Tim Ingold teve seu ensaio Against Soundscape publicado na revista Autumn Leaves: Sound and the Environment in Artistic Practices. O título foi suavizado quando ele o relançou em uma compilação de trabalhos seus, algum tempo depois: foi quando Contra a paisagem sonora transformou-se em Quatro objeções ao conceito de paisagem sonora (INGOLD, 2011), cujo corpo do texto foi mantido intacto. De forma sucinta e direta, o autor afirmou que, embora tenha contribuído para projetar o som como objeto dos mais variados estudos, o conceito de paisagem sonora sobrevive quando não tem mais utilidade e deve ser abandonado (INGOLD, 2011, p. 136).

O principal problema apontado por Ingold (2011) é que a paisagem sonora conduziria os novos estudos sobre cultura sônica ao erro que ele julga ter incorrido os estudos sobre 
cultura visual, que seria o de perder contato com os fenômenos do som, assim como teria sido perdida a conexão com os fenômenos da luz.

O 'visual', nesses estudos, parece nada ter a ver com o que significa ter capacidade de ver. Isso significa dizer que ele quase não lida com o fenômeno da luz. Ao invés disso, trata-se da relação entre objetos, imagens e suas interpretações. Um estudo sobre cultura aural, sustentada sobre as mesmas bases, seria sobre a interpretação de um mundo de coisas oferecidas em suas formas acústicas. (INGOLD, 2011, p. 136). ${ }^{3}$

Eis a primeira das quatro objeções anunciadas ao conceito de paisagem sonora: não haveria um mundo para cada sensório; perceberíamos o mesmo mundo com um arsenal de sensórios diferentes.

O poder do conceito prototípico de paisagem reside precisamente no fato de que ele não está atado a qualquer registro sensorial específico - seja visão, audição, tato, olfato, ou o que for. Na prática perceptual comum, esses registros cooperam com tamanha proximidade, e com tanta sobreposição de funções, que é praticamente impossível desenredar suas respectivas contribuições (INGOLD, 2011, p. 136). ${ }^{4}$

Ingold (2011) entende que a dimensão sonora dos lugares a que Schafer se refere na paisagem sonora não estaria para a paisagem como esta é entendida na pintura, mas para a paisagem como conceito geográfico, que diz respeito a uma comunidade habitando um mesmo lugar e as relações entre esse grupamento e esse espaço. Ari Y. Kelman, que exploraremos mais a seguir, também aponta que, apesar de ser compositor, Schafer está menos preocupado com a paisagem sonora como empreendimento estritamente artístico do que com posicioná-la num quadro em que se discute ordem social e ameaças ambientais (KELMAN, 2010, p. 220).

Segunda objeção de Ingold: assim como o poder da visão não residiria essencialmente em imagens, o poder da escuta não estaria essencializado em gravações. Ingold faz uma crítica à mediação tecnoartística que entende ser intrínseca ao conceito, como se uma paisagem sonora só existisse depois de ser processada por técnicas artísticas e/ou de gravação, para então ser reproduzida em ambientes preparados para tal (INGOLD, 2011, p. 136-137). Aqui ele capta uma contradição de Schafer em relação ao som gravado que será exposta mais detidamente a partir de outros autores nos próximos tópicos.

A terceira objeção é a de que o som não seria objeto da nossa percepção, e sim medium dela. Para o antropólogo, não escutamos o som. Em vez disso, escutamos coisas no som, por meio do som. O mesmo acontece em relação à luz, por meio da qual vemos coisas; e, no entanto, não vemos uma paisagem luminosa (lightscape), e sim uma paisagem banhada em luz. Por isso, paisagem sonora é uma ideia que faz pouco sentido para Ingold (2011).

A quarta e última objeção colocada por ele é que, em vez de pensar coisas em sua fixidez, a partir de superfícies que imaginamos rígidas, seria preciso pensar as fluências desse amplo medium que é onde existimos e por onde nos movemos (INGOLD, 2011, p. 138). Isso envolveria ventos, chuvas, sazonalidades. O ambiente, para Ingold (2011), deve ser pensado mais em relação à meteorologia do que aos estudos da paisagem, que estão mais próximos da escala humana e que talvez não levem em plena consideração outros movimentos complexos observáveis na escala do planeta. "[O] termo mundano pelo qual venho chamando os fluxos do medium é o clima” (INGOLD, 2011, p. 138, grifo do autor). ${ }^{5}$

O clima seria aquilo fundamental à percepção porque ele não é o que percebemos, mas aquilo em que percebemos. Se pensarmos com o autor que "[s]om e luz [...] são infusões do medium no qual encontramos nossa existência e pelo qual nos movemos” (INGOLD, 2011, p. 138) ${ }^{6}$, chegaremos à ideia de que é preciso pensar as fluências do medium como clima, 
ou seja, com muito mais variáveis e menos rigidez do que estamos acostumados a adotar em nossas pesquisas. Para colocar o fenômeno do som no centro das nossas investigações, o autor acredita que é necessário primeiro fazer uma pergunta que pode soar um tanto básica, mas que se apresenta incontornável: “o que é som?” (INGOLD, 2011, p. 137, grifo do autor). ${ }^{7}$

O texto de Ingold (2011) é rico em insights, em levantar questões que não estão desenvolvidas em Schafer, e acaba por abrir inúmeras possibilidades de debates que antes estavam travados por uma certa inércia decorrente da aceitação tácita de que paisagem sonora respondia a todas as questões. O ensaio de Ingold (2011) também instiga diversas contracríticas, o que não será feito aqui para não fugirmos do tema da paisagem sonora. Mas é importante salientar que o antropólogo continua debatendo a respeito do som ao longo de outros trabalhos, abrindo novas frentes de discussões.

Outro aspecto digno de menção é que Ingold (2011) não considera que a antropologia seja hoje uma disciplina estritamente social, por entender que é necessário envolver de forma mais ampla o ambiente em que vivem os humanos, não se limitando a eles. Para ele, diferenças culturais dizem respeito a diferenças de percepção - a isso se deve seu interesse pelo corpo e pelo ambiente. Seu foco nas materialidades do som e o envolvimento do conceito de medium em seus estudos o tornam um pesquisador com quem a Comunicação pode dialogar, especialmente aquela que também almeja extrapolar os limites do social.

\section{Um termo potente repleto de contradições internas}

Interessado pela vida social do som, Ari Y. Kelman (2010), professor de Estudos Americanos da Universidade da Califórnia, em Davis, admite que paisagem sonora é um termo fundamental para os estudos do som, mas também o considera problemático. Para ele, a formulação de Murray Schafer guarda sérias contradições internas, ainda que seja de uma potência inegável. Apesar de sua grande popularidade, a falta de rigor em sua aplicação também seria generalizada. Kelman (2010) analisou incongruências tanto na concepção original quanto em pesquisas posteriores, avaliando como estas últimas se relacionam ou deixam de se relacionar com a paisagem sonora concebida por Schafer.

Kelman (2010) observou que muitos estudiosos aproveitam a ressonância do termo, mas o adotam de maneira superficial, sem engajamento nas ideias do autor. Outros o redefinem ou o reenquadram conceitualmente, levando os leitores a reconsiderá-lo de forma mais detida. Este seria o caso de Barry Truax (2001) - com a ressalva de que a ideia de comunicação acústica de Truax acabaria reduzindo as possibilidades do som a uma estreita esfera ideal de interação humana, de modo habermasiano (KELMAN, 2010, p. 224-225).

Haveria também aqueles autores que, mesmo não fazendo qualquer referência à paisagem sonora, conseguiriam resultados satisfatórios em suas explorações sobre a dimensão sonora do ambiente. Um dos exemplos, citado tanto por Kelman (2010) quanto por Sophie Arkette (2004), é Alain Corbin. Em Village Bells, ele aborda o uso de sinos e sua mudança de status na vida política e cultural de vilarejos franceses. Utilizando a expressão paisagem auditiva, "[o] que ele descreve [...] não é de maneira alguma uma paisagem sonora nos termos de Schafer, mas algo mais complexo, mais rigoroso, mais sofisticado e detalhado" (KELMAN, 2010, p. 227). ${ }^{8}$

Um dos principais problemas que Kelman (2010) identifica em A Afinação do Mundo, de Schafer, é que o livro é muitas vezes tomado como um texto descritivo quando seria em grande parte prescritivo. A obra traz uma série de instruções sobre como o ambiente deve soar ou não, quais sons importam ou não, e como muitos desses sons deveriam ser silenciados ou nem mesmo postos em circulação. De viés ideológico, esse projeto também 
estaria na base das ideias de limpeza de ouvidos ${ }^{9}$ e projeto acústico ${ }^{10}$. Nesse sentido, a noção de paisagem sonora pareceria hermeneuticamente oca por estar limitada aos próprios preconceitos e preferências estéticas de Schafer (KELMAN, 2010, p. 230). "A paisagem sonora de Schafer não é, de maneira alguma, um campo de investigação aural neutro; pelo contrário, ela é profundamente informada pelas próprias preferências de Schafer por uns sons em detrimento de outros" (KELMAN, 2010, p. 214). ${ }^{11}$

Um desses preconceitos surgiria quando o canadense advogou que a paisagem sonora dita original era de maior quietude, mais silenciosa. Apesar de Schafer (2001) reiterar essa ideia ao longo de todo o livro A Afinação do Mundo, Kelman (2010) não se mostra convencido de que o autor tenha conseguido explicar se tal paisagem sonora tenha, de fato, alguma vez existido - e, neste caso, onde ou quando. De qualquer forma, Schafer (2001) constrói uma dicotomia entre rural e urbano, dissertando a favor do primeiro. O rural seria dotado de uma perspectiva sônica mais distinguível (e agradável a ele) do que o panorama acústico ruidoso e indistinto (para ele) das cidades industriais; é o que Arkette chama de preconceito com o urbano (ARKETTE, 2004, p. 161). Para ela, Schafer tapou os ouvidos para as potencialidades do som na compreensão das relações que se dão nas cidades. Não se trata de ser condescendente com abusos, afirma a pesquisadora - seria possível tratar deles sem rejeitar as características próprias desses lugares.

Schafer (2001) afirma que as paisagens sonoras urbanas são $l o-f i^{12}$, o que para ele significa que a diferenciação entre sinal e ruído é muito baixa. Dessa maneira, seria extremamente difícil identificar o que é informação ou não no contexto agitado das cidades. A crítica de Kelman (2010) a respeito desse aspecto, em particular, é que Schafer estaria, assim, desconsiderando as complexidades e particularidades dos contextos urbanos e subestimando as possibilidades de agência de seus habitantes. Seria como se os citadinos não pudessem desenvolver técnicas de escuta para lidar com seu próprio ambiente. "A análise de Schafer não leva em consideração o desenvolvimento de tais habilidades em meio ao alarido da vida urbana moderna porque, em sua formulação, todos os sinais [no contexto das cidades] foram reduzidos a ruído" (KELMAN, 2010, p. 217) ${ }^{13}$. Nesse sentido, a forma como Schafer concebe paisagem sonora seria demasiadamente determinista e totalizante.

Kelman (2010) também destaca contradições na noção de esquizofonia. Schafer refere-se a essa ideia como a

[...] separação entre o som original e sua reprodução eletroacústica. Os sons originais são ligados aos mecanismos que os produzem. Os sons reproduzidos por meios eletroacústicos são cópias e podem ser reapresentados em outros tempos e lugares. Emprego esta palavra nervosa para dramatizar o efeito aberrativo desse desenvolvimento do século XX. (SCHAFER, 2001, p. 364, grifo do autor).

Schafer (2001) entende que a dissociação entre o que chama de som natural e seu registro gravado para posterior reprodução (para ele, um som não natural) pode levar a sentimentos desnorteadores.

Tal divisão entre natural e não natural é questionável, ressalta Kelman (2010). Não seriam esses sons - gravados e reproduzidos - parte constitutiva da experiência sonora do ambiente? Se formos além, é possível indagar ainda sobre “[...] a irracionalidade da justaposição eletroacústica” (SCHAFER, 2001, p. 140). O que haveria de irracional nessas técnicas de gravação, edição e difusão de áudio? O que Schafer (2001) entende, aí, por irracionalidade - e, como consequência, por racionalidade - quando ele mesmo utilizou e incentivou o uso de gravação para o estudo de paisagens sonoras? É uma pergunta relevante 
se consideramos que Schafer realizou programas de rádio a partir de gravações ambientais e compôs música eletroacústica a partir desse tipo de registro.

Na avaliação de Kelman (2010), boa parte dessas estranhezas encontradas no pensamento de Schafer deve-se ao fato de o autor confundir som e escuta. A Afinação do Mundo sugere possibilidades de intervenção no espaço social para alcançar um ambiente sonoro mais equilibrado, segundo os próprios critérios de seus leitores mais entusiastas (o que sempre requer cuidado). Porém, o método que seu autor esboçou para a orquestração da paisagem sonora é a escuta: ouvir o ambiente como se fosse uma sinfonia de seu agrado, destacando o que importa. Isso não implicaria, para Kelman (2010), engajamento com a paisagem sonora. Em vez de ser um encontro com o ambiente acústico, tal postura seria uma sublimação dele - alcançada por meio de uma escuta treinada que seleciona o que interessa. "A paisagem sonora torna-se algo a ser superado, nem mesmo é algo a ser escutado" (KELMAN, 2010, p. 218). ${ }^{14}$

Assim, a noção schaferiana de paisagem sonora traria a reboque um certo desprezo pelo ruído de fundo, desconsiderando que é apenas em relação a ele que qualquer informação faz sentido. "A intenção original de Schafer - silenciar o ruído de fundo - parece estar em conflito com seu próprio legado, no qual o ruído de fundo não impede a produção de sentido, mas é intrínseco a ela" (KELMAN, 2010, p. 230). ${ }^{15}$

Uma postura diferente de a de desconectar o ruído de fundo dos sons que importariam seria tomá-lo como parte de um processo mais amplo, no qual se operam mudanças. Nesse sentido, Kelman (2010) traz ao debate a sugestão do etnomusicólogo Steven Feld de uma guinada da esquizofonia para a ideia de esquismogênese de Gregory Bateson, que poderia dar conta de diferenciações progressivas entre grupos sociais e indivíduos. Enquanto em Schafer a paisagem sonora seria um mero background sonoro para a vida social, Kelman (2010) destaca a proposta de Feld de encarar o som não como algo a ser reparado, mas a ser investigado. Essa mudança também exigiria uma atenção ao som, a suas qualidades, e não tanto às características da experiência da escuta.

Subestimado por Schafer (2001), o ruído de fundo seria, paradoxalmente, aquilo pelo que se interessa grande parte dos estudiosos de alguma forma influenciados por ele. Segundo Kelman (2010), muitos desses pesquisadores continuam, porém, a usar o termo paisagem sonora sem apontar retificações, sem expor e problematizar em que aspectos seus trabalhos se aproximam ou se distanciam das ideias originais de Schafer. A adesão às ideias parece acontecer, mas no fundo não é o que efetivamente ocorre. O termo paisagem sonora figura, apenas. Alguns trabalhos analisados por Kelman “[...] capitalizam a seu favor a ressonância da ideia de Schafer", mas "[...] fracassam em engajar-se na ideia de "paisagem sonora' para além da sua leitura mais superficial” (KELMAN, 2010, p. 223). ${ }^{16}$

Contudo, permanece uma dúvida: diante de todas essas inconsistências e vieses, a paisagem sonora ainda seria um termo útil para pesquisas? Para Kelman (2010), não se pode negligenciar seu potencial e a amplitude de sua difusão. Por outro lado, também não é mais possível utilizá-lo sem que se compreenda suas implicações, sem se ter a consciência do que o termo carrega em sua origem. Em suma, não se pode ignorar o pensamento de Schafer - e isso inclui os problemas intrínsecos a ele. Seria necessário encontrar um meio-termo frutífero, mas para isso também seria preciso exploração, definição e enquadramento adequado.

"Schafer moldou o campo [dos estudos do som] de uma maneira que excede sua própria contribuição” (KELMAN, 2010, p. 213) ${ }^{17}$, e o campo deve continuar buscando respostas sólidas às suas questões basilares. Para Kelman (2010), a primeira delas é o que é som (nisso, aproxima-se de Tim Ingold [2011]). A segunda, de caráter metodológico, indaga 
a respeito do modo como se reúne informação, quais são as teorias que guiam os estudos e quais os modelos existentes para a investigação do som.

\begin{abstract}
A vasta e escorregadia explicação de Schafer para paisagem sonora oferece pouco ou nenhum modelo executável para se estudar a vida social do som. O livro homônimo [The Soundscape, ou A Afinação do Mundo] é ora polêmico, ora amplamente historiográfico, e às vezes sonhadoramente teórico. Ele confunde som e escuta, está cheio de contradições estranhas, e carrega um forte viés contra música gravada de todos os tipos, mesmo que o próprio Schafer estivesse envolvido em realizar gravação e catalogação de som e estimulasse outros a fazerem o mesmo. No entanto, o termo ainda ressoa, e o faz muito bem para além dos limites do livro. (KELMAN, 2010, p. 228) ${ }^{18}$
\end{abstract}

\title{
Um conceito para o sujeito autocentrado da estereofonia
}

Autor bastante referenciado nas pesquisas sobre som, Jonathan Sterne vem desenvolvendo uma crítica à paisagem sonora que conta com interessantes reviravoltas. Em 2013, ele assinou um capítulo intitulado Soundscape, Landscape, Escape no quinto volume da coleção Sound Studies, Soundscapes of the Urban Past. A colaboração - a que Sterne (2013) se refere como uma "curta meditação" - aparenta ter sido concluída apressadamente, pois faltam não apenas detalhes (como trazer na bibliografia alguns livros citados no corpo do escrito), mas até mesmo desenvolvimentos inteiros que são anunciados de início. Sterne ali se dispôs a realizar "[...] uma história bastante esquemática da palavra paisagem sonora, e uma leitura dela a partir da perspectiva da assim chamada 'virada espacial' nas humanidades e ciências sociais” (STERNE, 2013, p. 183, grifo do autor) ${ }^{19}$. Contra as expectativas, o que acontece ao longo das páginas assemelha-se mais a um esboço da primeira tarefa e à expressão de um desejo, no caso da segunda.

Eis que, dois anos depois, o trabalho ressurge diferente, como capítulo do livro Living Stereo: Histories and Cultures of Multichannel Sound ${ }^{20}$ (STERNE, 2015). Não se trata do mesmo texto e tampouco deixa de ser o anterior. Quase a totalidade da primeira versão foi mantida, com alguns trechos mudando de lugar e a bibliografia faltante sendo devidamente listada. Poucas coisas foram subtraídas. Em grande parte, o autor desenvolve e aprofunda as ideias que havia introduzido, trazendo informações novas 21 . Sterne (2015) afirma que falta uma história intelectual mais completa do termo paisagem sonora, mas se tem acesso a uma substanciosa introdução ao tópico nessa segunda versão - que leva o título The Stereophonic Spaces of Soundscape ${ }^{22}$.

A paisagem sonora, para Sterne (2015), é um neologismo imediatamente acessível que serve de animador ponto de entrada a novatos no campo. Altamente ubíqua enquanto conceito, ela seria concebida de forma a implicar tanto um ponto de audição (centrado no sujeito) quanto uma espécie de onisciência. Para o autor, a quase ubiquidade do termo se deve à forma globalizante como Schafer definiu paisagem sonora:

Paisagem sonora-O ambiente sonoro. Tecnicamente, qualquer porção do ambiente sonoro vista como um campo de estudos. O termo pode referir-se a ambientes reais ou a construções abstratas, como composições musicais e montagens de fitas, em particular quando consideradas como um ambiente (SCHAFER, 2001, p. 366).

Paisagem sonora seria, portanto, o ambiente físico em sua totalidade, mas também uma porção dele entendida como campo de estudo. Paisagem sonora seria tanto a técnica de gravação e montagem como também seria a própria obra. Sterne afirma que Schafer usou paisagem sonora como um conceito social total, anunciando: “[...] desafio a noção amplamente 
aceita de que a paisagem sonora é tanto um espaço físico quanto sua representação" (STERNE, 2015, p. 67 ${ }^{23}$.

Para isso, o autor desenvolve o argumento de Stefan Helmreich, de que a ideia de paisagem sonora está intimamente ligada ao espaço estereofônico do som gravado que emerge no pós-guerra, muito embora a concepção schaferiana carregue uma política antimodernista que se oferece como uma espécie de saída à cultura do consumismo. Para Sterne (2015), essa contradição encontra-se escancarada nas ideias de paisagens sonoras hi-fi e lo-fi (respectivamente, de alta fidelidade e de baixa fidelidade). Isso porque, para compor um ambiente de alta fidelidade, é preciso a aplicação de uma série de técnicas, o uso diversificado de equipamentos e ambientes projetados a partir de um conjunto de ideias ligadas ao design acústico. A fim de discutir isso, Sterne traz a noção de audioposition (que traduziremos como audioposição), utilizada por Neal Verma

[...] para indicar o lugar do ouvinte, ao qual se chega codificando primeiros planos e planos de fundo. Com esse termo, a ideia também fica disponível como verbo, enfatizando que ele é sempre fabricado. Ouvintes não 'possuem' meramente uma áudio-posição; eles são 'posicionados' pela composição de áudio (VERMA, 2012, p. 35 apud STERNE, 2015, p. 70) ${ }^{24}$.

Assim como Ingold (2011) e Kelman (2010), Sterne (2015) entende que o termo paisagem sonora não está ligado, em primeira instância, ao campo das artes. Diferente disso, “[...] conceitos de paisagem sonora emergem de um conjunto mais amplo de discursos profissionais e estéticos que concebem o espaço sônico". (STERNE, 2015, p. 67) ${ }^{25}$. Compartilhariam, portanto, de perspectivas surgidas em campos que sua concepção original vai criticar ostensivamente.

Sterne alinha-se aos próprios editores de Living Stereo, Théberge, Devine e Everrett (2015), que tratam a estereofonia - e suas promessas de realismo, alta definição, espacialidade e presença - dentro de um quadro conceitual em que ela é entendida não apenas como uma tecnologia, mas como um fenômeno estético que vem se alterando incessantemente ao longo de processos históricos e culturais iniciados ainda no século XIX. De lá para cá, não apenas as ciências acústicas teriam se constituído enquanto formação epistemológica, como também teria havido uma transição paradigmática do som e da escuta como fenômenos não espaciais para fenômenos fundamentalmente espaciais.

"Pensamos estereofonia como um conjunto de relações entre tecnologias de áudio, espaços acústicos (físicos e virtuais), técnicas de escuta, discursos científicos e comerciais, condições econômicas e contextos de recepção" (THÉBERGE; DEVINE; EVERRETT, 2015, p. 3). ${ }^{26} \mathrm{Tal}$ fenômeno teria produzido um sujeito ouvinte particular, uma vez que as ciências acústicas, enquanto constelação de disciplinas que promoveram abordagens físicas, fisiológicas, psicológicas e musicais em relação ao som, operaram processos de objetivação e subjetivação semelhantes ao que Foucault aponta em sua arqueologia das ciências humanas (THÉBERGE; DEVINE; EVERRETT, 2015, p. 17). Esse sujeito acústico criado pela estereofonia é fundamentalmente estático; precisa de uma posição ideal em um campo sonoro espacializado para poder ouvir aquilo que foi preparado para ele. A perspectiva auditiva é pensada e praticada como um sentido de direcionalidade sônica que trata de apagar suas marcas da experiência da escuta em favor de uma ilusão imersiva de alta fidelidade.

Para dar conta da história dessa subjetividade, seria preciso dar atenção, por exemplo, a fatores geracionais, étnicos e de gênero. Estudos como os de Keir Keightley e Tim Anderson, citados pelo trio de editores de Living Stereo (THÉBERGE; DEVINE; EVERRETT, 2015) e também por Sterne (2015), fazem uma ligação entre a cultura estereofônica e o 
escapismo de homens de classe média em ambientes domésticos a partir do surgimento dos home stereos nos Estados Unidos dos anos 1960. "Publicações da época alardeavam a explosão do hi-fi como uma rejeição dos gostos femininizados das massas, corporificados pela televisão [...]. Revistas e anúncios apresentavam o hi-fi como civilizado, sofisticado e edificante" (STERNE, 2015, p. 76). ${ }^{27}$

A morfologia da paisagem sonora de Schafer viria da estética das gravações hi-fi e dos sistemas de alta fidelidade na sala de estar dessa classe média. Para Sterne (2015), é esse sujeito autocentrado - e eventualmente escapista - da estereofonia que aparece como pré-condição da paisagem sonora.

Em uma paisagem sonora lo-fi, os sinais acústicos individuais são obscurecidos em uma população de sons superdensa. O som translúcido - passos na neve, um sino de igreja cruzando o vale ou a fuga precipitada de um animal no cerrado - é mascarado pela ampla faixa de ruído. Perde-se a perspectiva. (SCHAFER, 2001, p. 71-72).

Sterne comenta o trecho acima de A Afinação do Mundo (SCHAFER, 2001):

Perde-se a perspectiva. Aqui, a interdependência entre o conceito de paisagem sonora e um tipo de áudio-posição começa a se tornar clara. A paisagem sonora requer um sujeito ouvinte singular, unificado e coerente para ouvi-la, para apreendê-la, para criticá-la, para moldá-la, para transformá-la. O sujeito da paisagem sonora precisa de uma base estável. Qualquer outra coisa é uma potencial crise. (STERNE, 2015, p. 76, grifo do autor). ${ }^{28}$

Como artefato da cultura hi-fi, a paisagem sonora traria um apelo “[...] diretamente ligado às segundas naturezas - as posições sociais e disposições, o habitus - que abastecem os sistemas hi-fi e as salas de concerto invocados nos escritos de Schafer" (STERNE, 2015, p. 78). ${ }^{29}$ Sua proposta almejaria uma totalidade, uma onisciência, mas a transcendência que a paisagem sonora ofereceria seria uma que é datada e situada, firmada nos discursos audiotécnicos da estereofonia. Mesmo que servisse de plataforma para uma crítica em relação ao ambiente sonoro, mais particularmente à dimensão acústica das cidades, a paisagem sonora faria isso a partir de uma perspectiva auditiva dominante.

\section{Um conceito enraizado no espaço euclidiano}

É em proximidade com o que Henri Lefebvre (1991) entende como espaço dominante que Sterne (2001) situa a paisagem sonora. O filósofo e sociólogo francês concebe o espaço como algo socialmente produzido, engrossando as críticas ao espaço euclidiano, uma ideia de espaço como fundo inerte onde todo o resto acontece. Para pensar a produção do espaço, Lefebvre (1991) desenvolveu uma tríade conceitual composta pelas noções de práticas espaciais, representações do espaço e espaços representacionais. As representações do espaço são o espaço conceitualizado: “[...] o espaço de cientistas, planejadores, urbanistas, tecnocratas fragmentadores e engenheiros sociais, assim como um certo tipo de artista inclinado à cientificidade - todos os quais identificam o que é vivido e o que é percebido com o que é concebido" (LEFEBVRE, 1991, p. 38). ${ }^{30}$

Parte constituinte do processo de produção do espaço, o espaço concebido não pode ser tomado como o espaço em sua totalidade. O espaço social excede as representações do espaço, que são "o espaço dominante em qualquer sociedade (ou modo de produção). Concepções do espaço tendem, com certas exceções [...], a um sistema de sinais verbais (e, portanto, intelectualmente elaborados)” (LEFEBVRE, 1991, p. 38-39) ${ }^{31}$. 
Em sua tarefa de realizar uma breve genealogia do termo paisagem sonora, Sterne recuperou o seguinte trecho de uma das primeiras formulações a aparecer na obra de Murray Schafer, datando de dez anos antes do lançamento de The Tuning of the World:

Cada peça musical é uma elaborada paisagem sonora que poderia ser plotada em um espaço acústico tridimensional.

Falar de uma paisagem sonora, é claro, não é de forma alguma evocar música programática $^{32}$. Há uma diferença entre falar sobre espaço e tentar preencher aquele espaço com objetos. $O$ espaço ao qual me refiro é vazio, a não ser pelos sons que o atravessam.

Não há 'país’33 em uma paisagem sonora. (SCHAFER, 1967, p. 13 apud STERNE, 2015, p. $71-72)^{34}$.

Da citação, que aparece na já mencionada publicação Ear Cleaning, Sterne (2015) destaca a frase em que Schafer associa a paisagem sonora a um espaço vazio onde a única coisa que há são os sons. Essa imaginação do espaço é, para Sterne (2015), a de um espaço euclidiano clássico, demasiadamente desprovido de relações. O som não se propaga em um espaço assim: ele só existe conformado pelo próprio espaço onde ressoa. A partir do pensamento da virada espacial, imaginar a paisagem sonora com base nessa ideia de espaço limita-a consideravelmente enquanto conceito.

Sterne (2015) levanta usos do termo paisagem sonora que antecedem o de Schafer. Em uma entrevista concedida a Carlotta Darò (uma das pesquisadoras que muniram Sterne de dados complementares entre as duas versões de seu trabalho aqui analisadas) (DARÒ, 2012, p. 185, apud STERNE, 2015, p. 186), Schafer declarou que a palavra soundscape veio de uma série de palestras e ensaios do geógrafo e planejador urbano Michael Southworth. Apoiando-se nas investigações de Darò e de Sabine von Fischer, assim como na leitura do ensaio The Sonic Environment of Cities, resultado de trabalho de campo de Southworth sobre "A percepção da paisagem sonora de Boston", publicado em 1969, Sterne relata:

Seu conceito de paisagem sonora foi tomado de seu orientador no MIT, Kevin Lynch, que usou cityscape para denotar a facilidade com a qual partes da imagem de uma cidade podiam ser apreendidas. Southworth estava procurando por um aspecto da vida da cidade que não tivesse ainda sido considerado no modelo de Lynch, então ele se voltou para outros sentidos, rejeitando tato e cheiro por suas efemeridades, e voltando-se para o som. Southworth era altamente influenciado pelo novo urbanismo de autores como Jane Jacobs, e suas críticas à abordagem modernista à arquitetura e ao planejamento urbano. [...] As conclusões de Southworth antecipam bem alguns dos pontos mais conhecidos de Schafer: apreensão é um problema para o som por causa do ruído urbano, que ele considera principal obstáculo ao projeto sônico em cidades [...]. [Southworth] argumenta que o alto contraste entre sons de primeiro plano e de plano de fundo fazem os espaços sonoros mais identificáveis; e que espaço aberto e espaços responsivos guardam um potencial especial para experimentação e para encenar eventos sônicos. Finalmente, ele aponta para os sinais sônicos (o que Schafer chamaria de marcas sonoras) como uma chave para experiências sonoras nítidas da cidade. (STERNE, 2015, p. 70-71)..$^{35}$

Southworth criou a expressão sinais sônicos (sonic signs), adaptando a terminologia desenvolvida pelo seu orientador, Kevin Lynch (STERNE, 2015). Autor de A Imagem da Cidade (1997), Lynch relaciona os elementos urbanos para poder trabalhar a visibilidade deles e, dentre esses elementos, estão os marcos (landmarks). Com o termo marcas sonoras (soundmarks), Schafer alteraria a nomenclatura de Lynch de forma muito menos sutil do que havia feito Southworth. 
Como Sterne (2015) observa, o que parece haver com os neologismos de Schafer é uma inversão, para o sonoro, de vieses visuais usados na descrição do espaço, mas daí a fazer ecoar com alguma fidelidade o pensamento de um planejamento urbano crítico haveria alguma distância; no que Murray Schafer se assemelha a Jane Jacobs, que escreve sobre o comum e o cotidiano, "[...] sobre o funcionamento das cidades na prática, porque esta é única maneira de saber que princípios de planejamento e que iniciativas de reurbanização conseguem promover a vitalidade socioeconômica nas cidades e quais práticas e princípios a inviabilizam" (JACOBS, 2000, p. 1-2)?

Jacobs (2000) criticou a ideia de Cidade-Jardim, que se difundiu como uma alternativa à cidade mas acabou resultando na destruição das qualidades que são próprias aos espaços urbanos. Ela atacou o desprezo de Lewis Mumford pela centralidade fervilhante das cidades antigas e a ideia de Le Corbusier de criar uma cidade que seria inteiramente um parque, simplesmente se construindo arranha-céus no meio de amplas áreas verdes. Onde Schafer se encaixa nesses embates entre modos de pensar a cidade? Jacobs levantase contra cânones - cabe-nos perguntar o que faz Schafer dentro de sua própria tradição e como tudo isso se relaciona à dinâmica de outras tradições das quais ele se aproxima.

Considerando que investigações sobre essa relação entre a gênese da paisagem sonora de Schafer e expoentes do planejamento urbano estão apenas começando, não podemos apressar-nos e simplesmente inverter as noções de um campo para outro, tomando a vertente modernista na arquitetura e seus desdobramentos no planejamento urbano como equivalentes à música moderna. Análises mais detidas e aprofundadas devem seguir o levantamento desses dados, agora melhor compilados, sobre a genealogia do termo paisagem sonora. Mas é importante compreender que Schafer o concebeu a partir de um pensamento composicional ligado à música ocidental de concerto do século XX - “[...] de 4'33” de John Cage à musique concrète de Pierre Schaeffer, da música eletrônica à tape music" (STERNE, 2015, p. 73).$^{36}$ Se por um lado Schafer foi, para Sterne, inovador em relação à sua linhagem de compositores ao pensar que composições poderiam criar espaço; por outro, manteve o modelo heroico do compositor - desta vez na figura do engenheiro acústico.

\section{Um conceito de mídia}

Tim Ingold (2011) defende que o uso da paisagem sonora em pesquisas deve ser abandonado, mas se refere à ideia como conceito. Já Ari Y. Kelman (2010) afirma que ela é fértil e ainda pode ser útil, mas não vai além de tomá-la como um termo. Na primeira versão de seu trabalho, Sterne (2013) pensava paisagem sonora enquanto constructo. "Uso constructo ao invés de conceito para observar que a teoria da paisagem sonora também guarda relações com práticas culturais particulares, e para destacar que não existe uma única e coerente concepção de paisagem sonora que seja consenso entre estudiosos e praticantes" (STERNE, 2013, p. 183, grifo do autor) $)^{37}$.

Eis uma das poucas coisas subtraídas entre uma versão e outra. Sterne (2015) abre mão do entendimento de constructo na segunda versão publicada em favor da compreensão de paisagem sonora não apenas como um conceito, mas como um conceito de mídia. É possível encontrar um dos fundamentos dessa mudança quando Sterne declara que Cage e Karl Stockhausen (de quem Schafer teria herdado o modo de abordar composição como espaço) eram compositores que desenvolveram suas próprias teorias de mídia (STERNE, 2015, p. 74). Isso se dá porque lidaram com as mais variadas mídias técnicas no curso de seus trabalhos composicionais e escreveram sobre suas práticas. 
Há outra base para uma associação mais direta de paisagem sonora com os estudos de mídia. Sterne teve acesso a uma carta que Murray Schafer remeteu a Marshall McLuhan em 1974. Na correspondência, o remetente admite ter sido influenciado pela leitura dos trabalhos do destinatário, mas critica a capacidade do autor de A Galáxia de Gutemberg (1977) de empreender uma exploração sobre a mudança "[do modo visual] para o modo auditivo do homem eletrônico" (MCLUHAN, 1977, p. 105): “[...] para alguém que acredita que estamos implodindo de volta a uma era aural-tribal, seu conhecimento sobre assuntos relativos à audição sempre pareceu muito escasso" (SCHAFER, 1974 apud STERNE, 2015, p. 75$)^{38}$. No documento, que integra o corpus da pesquisa de Olga Touloumi sobre a história da ideia mcluhaniana de espaço acústico, Schafer explica que "[p]or assuntos relativos à audição, não estou apenas me referindo à história da música, mas a todo o desenvolvimento do ambiente acústico - que eu chamo de paisagem sonora” (SCHAFER, 1974 apud STERNE, 2015, p. 75) 39 .

Para Sterne (2015), é quando Schafer pensa a paisagem sonora como hi-fi ou lo-fi que ele estaria explicitamente a concebendo como um sistema de reprodução e transmissão sonora. "[P]aisagem sonora é um conceito de mídia, e é um conceito que demanda que seu ouvinte tenha a experiência do amplo fenômeno de mediações sônicas a partir de uma posição estável e surpreendentemente delicada" (STERNE, 2015, p. 67) ${ }^{40}$. Em vez de servir de plataforma para uma crítica ao fenômeno de mídia, como vem sendo compreendida, a paisagem sonora seria, para Sterne (2015), uma definição de mídia em seu negativo, ou seja, alternativa a 1) uma subjetividade integrada; 2) uma totalidade; e 3) uma possibilidade de perspectiva - sendo todas essas ideias almejadas no ideal de Schafer (2001).

O problema maior não seria nem mesmo o equívoco, expresso na noção de esquizofonia, de acusar a mídia de separar o som de sua fonte. Isso sequer faria sentido se considerarmos que todos os sons só existem apartados de suas fontes (STANYEK; PIEKUT, 2010 apud STERNE, 2015, p. 68). A questão é anterior: enquanto Schafer atribui efeitos desestabilizadores a tecnologias como o telefone, o fonógrafo e o rádio, parece não se dar conta de que "[...] as margens do seu sujeito ouvinte bem centrado já estão perturbadas e moldadas pelos meios" (STERNE, 2015, p. 68). ${ }^{41}$

Sterne (2015) toma emprestadas algumas reflexões de Friedrich Kittler e John Mowitt sobre o filósofo alemão Martin Heidegger. Segundo essas elucubrações, de alguma forma, Heidegger creditava à relação que manteve com o rádio à própria emergência de sua noção de Dasein. Tecnologias como o rádio possuiriam uma função de enquadramento do mundo, tornando-o acessível aos ouvidos humanos. Tal ideia conectar-se-ia à noção de audioposição. "A essência da paisagem sonora, e de fato a essência do estéreo, não é o espaço físico ou a relação entre o espaço físico e sua representação. Sua essência é uma audioposição estática, uma a partir da qual o mundo inteiro está disponível para ser ouvido" (STERNE, 2015, p. 79, grifo do autor) ${ }^{42}$. Se assim for, será que estamos tomando o ambiente pelas concepções limitadas, enquadradas e enviesadas que criamos para ele?

\section{Considerações finais: um conceito de partida}

Ao longo deste artigo, buscamos apresentar e articular questionamentos dirigidos à ideia de paisagem sonora que vêm surgindo nos últimos anos. Acompanhamos, inclusive, como o pensamento de um mesmo estudioso pôde mudar em pouco tempo - o que revela uma grande vitalidade do debate na atualidade. Algumas questões sobressaem - uma das mais desafiadoras talvez seja a necessidade de pensarmos sobre aquilo a que nos referimos quando mencionamos paisagem sonora, mas também sobre o que viria a ser o próprio som. 
Estamos todos falando da mesma coisa? Ou será que, quando começamos a nos debruçar sobre o que entendemos por som, veremos emergir diferenças na maneira como concebemos, por exemplo, o entrelaçamento entre ouvinte e ambiente? Se sim, com essas diferenças, é provável que venha a tomar relevo todo um conjunto de discussões, como as que existem sobre a relação entre sujeito e objeto.

Mais particularmente sobre paisagem sonora, percebemos que hoje o termo é amplamente utilizado nos mais diversos âmbitos, dentro e fora da academia e, como não poderia deixar de ser, também na música e nas demais artes sonoras. No entanto, apesar de Murray Schafer, autor da concepção de paisagem sonora que acabou por se popularizar, ser ele mesmo um compositor, a ideia está impregnada de problemáticas trazidas de outros campos disciplinares desde sua origem. Schafer (1974 apud STERNE, 2015) criticou Marshall McLuhan por este não ter conhecimento suficiente sobre som para tratar dele com a propriedade necessária para o desenvolvimento de uma ideia como espaço acústico. Teria o compositor, por sua vez, cometido equívoco semelhante ao servir-se de um conceito como paisagem, por exemplo?

Com base em análises de suas obras e em dados como cartas e entrevistas, observamos que a gênese da ideia de paisagem sonora se desenvolve a partir de uma relação de Murray Schafer com autores de outras áreas - não apenas com McLuhan, dos estudos de mídia, mas também com Michael Southworth, da Geografia e do Planejamento Urbano. No entanto, Schafer parece não se ter aproximado das discussões epistemológicas desses campos. Isso pode ser o motivo de ele não ter estado atento às necessidades de se pensar um espaço para além de uma concepção euclidiana, inerte, carente de relações. Também pode não ter incorporado discussões menos apocalípticas (parafraseando Umberto Eco) sobre as mudanças que os meios técnicos contemporâneos podem operar na cultura. Esses pontos, ainda que pouco trabalhados, são parte constitutiva de sua difundida concepção de paisagem sonora, e devemos dar atenção a eles.

Diante de todas as considerações aqui expostas, é provável que uma pergunta de ordem mais imediata se levante. O que fazer em relação à paisagem sonora em nossas pesquisas sobre o som: adotá-la ou não? Pensamos, no entanto, que a questão não se trata de um. “tudo ou nada”. Quando Tim Ingold (2011) sugere o abandono do conceito, ele faz isso construindo objeções a partir de suas fragilidades. A paisagem sonora ainda está lá, orientando o debate, ainda que em direção a outros entendimentos. Não há como ignorar, hoje, os efeitos de sua popularidade; é preciso saber dialogar com a produção acadêmica que considera válida a abordagem da paisagem sonora - como a de pesquisadores como Emily Thompson, Barry Truax, Kay Kaufman Shelemay, David W. Samuels, Louise Meintjes, Ana Maria Ochoa, Thomas Porcello, se ficarmos só em autores citados nos trabalhos aqui discutidos.

Por outro lado, nossa posição tende a ser de discordância em relação a Sterne quando ele afirma que as inconsistências e contradições apontadas na paisagem sonora são um problema apenas para aqueles que não compartilham das convicções políticas e estéticas de Schafer em função deste nunca ter escondido suas fontes e posicionamentos (STERNE, 2015, p. 67). Mesmo aqueles que, sim, pensam em consonância com Schafer não podem usar paisagem sonora para tudo, valendo-se do gesto do autor de tentar cobrir toda a questão sonora com um conceito pretensamente total, sob a pena de inviabilizar a própria paisagem sonora como conceito. $\mathrm{O}$ que aparentemente serve a tudo acaba por perder força conceitual, em termos científicos. É por isso que, embora permaneça um termo útil para muitos, a paisagem sonora "[...] levanta a questão do espaço, escala e cultura sem nos oferecer as ferramentas para respondê-la” (STERNE, 2015, p. 78) ${ }^{43}$. 
Se estamos empenhados em rupturas epistemológicas para seguirmos com os estudos sobre o som, é preciso nos voltarmos para uma avaliação menos condescendente dos textos que fundam o campo, encarando problemáticas que estão impregnadas em sua própria terminologia. Paisagem sonora não é conceito final. Ela está mais próxima de um conceito de partida para discussões que tendem a ser - ou pelo menos deveriam - cada vez mais complexas e aprofundadas.

\section{Notas}

1 A primeira tradução de A Afinação do Mundo lançada no Brasil, em 2001, refere-se ao livro de 1993, The Soundscape, e tem como subtítulo em português Uma exploração pioneira pela história passada e pelo atual estado do mais negligenciado aspecto do nosso ambiente: a paisagem sonora.

2 Este é apenas o primeiro de três casos de mudanças de títulos em obras que citaremos neste trabalho. Apesar de poder ser um pouco maçante para os leitores, decidimos traçar essas alterações por dois motivos importantes. Em primeiro lugar, mudanças nos nomes dos trabalhos podem sugerir variações no pensamento dos próprios autores. Em segundo lugar, ao destrinchar abertamente o nome das obras - apontando que dois títulos diferentes se referem a duas edições do mesmo trabalho, ou ainda que um autor em determinado momento se engana sobre o título de um trabalho que ele mesmo escreveu - pode poupar o tempo de quem venha a buscar tais referências, além de minimizar potenciais mal-entendidos em debates futuros. Pelo esforço de síntese, em alguns momentos a leitura pode se tornar particularmente densa, em face ao volume de informações apresentadas e articuladas entre si, mas justificamo-nos com o entendimento de que é importante trazer ao debate novas pesquisas que possam acrescentar modos possíveis de pensar o entorno sonoro em nossas investigações.

3 "The 'visual', in these studies, appears to have nothing to do with what it means to be able to see. That is to say, it scarcely deals with the phenomenon of light. It is rather about the relations between objects, images and their interpretations. A study of aural culture, built along the same lines, would be about the interpretation of a world of things rendered in their acoustic forms." Optamos por trazer os trechos das citações em seus idiomas originais, observando que todas as traduções do inglês são nossas.

4 "The power of the prototypical concept of landscape lies precisely in the fact that it is not tied to any specific sensory register - whether of vision, hearing, touch, taste or smell. In ordinary perceptual practice these registers cooperate so closely, and with such overlap of function, that their respective contributions are impossible to tease apart."

5 "[T] $]$ he mundane term for what I have called the fluxes of the medium is weather."

6 "Sound and light (...) are infusions of the medium in which we find our being and through which we move".

7 "[W]hat is sound?"

8 "What he describes (...) is not a soundscape at all, in Schafer's terms, but is something more complex, more rigorous, more sophisticated and detailed."

9 “Um programa sistemático para treinar os ouvidos a escutarem de maneira mais discriminada os sons, em especial os do ambiente.” (SCHAFER, 2001, p. 365)

10 "Nova interdisciplina que requer os talentos de cientistas, cientistas sociais e artistas (em particular, músicos), o projeto acústico procura descobrir princípios pelos quais a qualidade estética do ambiente acústico, ou PAISAGEM SONORA, pode ser melhorado. Para isso é necessário conceber a paisagem sonora como uma vasta composição musical que ressoa incessantemente à nossa volta e perguntar de que modo sua orquestração e sua forma podem ser aperfeiçoadas para produzir riqueza e diversidade de efeitos que não sejam, todavia, destrutivos para a saúde ou o bem-estar humano. Os princípios do projeto acústico podem, assim, incluir a eliminação ou a restrição de certos sons (redução do ruído), a avaliação de novos sons, antes que eles sejam colocados indiscriminadamente no ambiente, mas também a preservação de sons (MARCOS SONOROS) e, acima de tudo, o arranjo imaginativo de sons para criar ambientes acústicos atrativos e estimulantes para o futuro. O projeto acústico pode também incluir a composição de ambientes-modelo, e nesse aspecto ele se aproxima da composição musical contemporânea. Comparar com ECOLOGIA ACÚSTICA.” (SCHAFER, 2010, p. 366, maiúsculas no original).

11 "Schafer's soundscape is not a neutral field of aural investigation at all; rather, it is deeply informed by Schafer's own preferences for certain sounds over others."

12 De baixa definição, ao contrário das paisagens sonoras rurais, que seriam hi-fi (de alta definição).

13 "Schafer's analysis cannot allow for the development of such skills amidst the din of modern urban life because in his formulation, all signals have been reduced to noise."

14 "The soundscape becomes something to be overcome, not even something to be heard."

15 "Schafer's original intention - to silence background noise - seems at odds with his own legacy in which background noise does not prevent the production of meaning, but becomes intrinsic to it."

16 "While the titles capitalize on the resonance of Schafer's idea, the books fail to engage the idea of the 'soundscape' beyond its most superficial reading." 
17 "Schafer shaped the field [Sound Studies] in ways that exceeded his own contribution."

18 "Schafer's vast and slippery explanation of the soundscape offers little or no workable model for studying the social life of sound. The eponymous book is at times polemical, at times broadly historiographic, and at times dreamily theoretical. It confuses sound and listening, it is rife with odd contradictions, and it bears a strong bias against recorded music of all kinds, even though Schafer himself was involved in and urged others to pursue the recording and cataloguing of sound. Yet, the term still resonates, and it does so well beyond the bounds of the book." 19 " [...] a very schematic history of the word soundscape, and reading it from the perspective of the so-called "spatial turn' in the humanities and social sciences."

20 Para o autor, o texto mais novo se trata da expansão das ideias daquele originalmente publicado na coleção Sound Studies. Há, no entanto, uma pequena confusão que convém esclarecer para que os leitores possam se assegurar de que estamos tratando dos mesmos textos: na primeira nota de rodapé do texto de 2015, Sterne refere-se à versão anterior, de 2013, como Escape from Soundscape, mas ela de fato foi publicada com o título Soundscape, Landscape, Escape.

21 Tais dados advêm de pesquisas de Carlotta Darò, Sabine von Fischer e Olga Touloumi.

22 De agora em diante, faremos referência a este texto consolidado de 2015, retomando o artigo de 2013 apenas quando discutirmos o que foi deixado de lado entre um e outro.

23 "I challenge the widely accepted notion that soundscape is both a physical space and its representation."

24 "I will use 'audioposition' to indicate the place of the listener that is carried by coding foregrounds and backgrounds. This term also seeks to make the idea available as a verb, stressing that it is always fabricated. Listeners do not just 'have' a point of audition; they are 'positioned' by audio composition."

25 "[C]oncepts of soundscape emerge from a wider set of professional and aesthetic discourses that conceive of sonic space."

26 "We think of stereophony as a set of relations between audio technologies, acoustic spaces (physical and virtual), listening techniques, scientific and commercial discourses, economic conditions and reception contexts."

27 "Periodicals of the time trumpeted the hi-fi boom as a rejection of the mass, feminized tastes embodied by television (...). Magazines and advertisements presented hi-fi as cultivated, sophisticated and edifying."

28 "Perspective is lost. Here, the interdependence of the soundscape concept and a kind of audioposition begins to become clear. The soundscape requires a coherent, unified, singular listening subject to hear it, to apprehend it, to criticize it, to shape it, to transform it. The subject of soundscape needs stable footing. Anything else is a potential crisis."

29 "That appeal is directly connected to the second natures - the social positions and dispositions, the habitus - that fill up the hi-fi systems and concert halls invoked in Schafer's writings."

30 "Representations of space: conceptualized, the space of the scientists, planners, urbanists, technocratic subdividers and social engineers, as of a certain type of artist with a scientific bent - all of whom identify what is lived and what is perceived with what is conceived."

31 "This is the dominant space in any society (or mode of production). Conceptions of space tend, with certain exceptions [...], towards a system of verbal (and therefore intellectually worked out) signs."

32 A música programática (program music) é aquela que se refere a narrativas ou imagens externas a si.

33 Poderíamos pensar que a tradução mais adequada para a palavra inglesa land, como no original, seria terra. No entanto, ao traduzi-la para o português, optamos por nos basear no radical latino pays, que na França medieval significava tanto habitante quanto território. Fizemos esta escolha porque há uma distinção entre paisagem como conceito geográfico e o sentido renascentista do termo. Segundo Verter Cozer (1999), a palavra portuguesa paisagem e suas variações em outras línguas latinas aparecem apenas no contexto da Renascença, já associadas às artes plásticas. Embora também possa referir-se à pintura, como um "espaço que se observa de um golpe de vista” (HOLZER, 1999, p. 152), o termo em inglês landscape, enquanto conceito geográfico, vem da palavra holandesa landschap, equivalente à alemã Landschaft, ambas de origem medieval. "Landschaft refere-se a uma associação entre o sítio e os seus habitantes, ou se preferirmos, de uma associação morfológica e cultural. Talvez tenha surgido de Land schaffen, ou seja, criar a terra, produzir a terra” (HOLZER, 1999, p. 152). O conceito geográfico de paisagem, portanto, pouco tem a ver com o sentido renascentista, estando voltado ao "significado medieval de área fisicamente e culturalmente reconhecível” (HOLZER, 1999, p. 153). Como os autores debatidos neste trabalho entendem que a noção de paisagem sonora de Schafer está mais próxima do conceito geográfico, trouxemos para a tradução o termo país, cujo radical latino nos parece mais relacionado ao sentido de land como em Land schaffen.

34 "Every piece of music is an elaborate soundscape which could be plotted in three-dimensional acoustic space. To speak of a soundscape, of course, is in no way to invoke program music. There is a difference in talking about space and attempting to fill that space with objects. The space to which we refer is empty save for the sounds cutting through it. There is no 'land' in a soundscape."

35 "His concept of soundscape was taken from his supervisor at MIT, Kevin Lynch, who used cityscape to denote the ease with which the parts of the image of a city could be apprehended. Southworth was looking for an aspect of city life that had not been considered yet in Lynch's model, so he turned to other senses, rejecting touch and smell for their ephemerality, and turning to sound. Southworth was heavily influenced by the new urbanism of authors like Jane Jacobs, and their critique of modernist approaches to architecture and urban planning. (...) Southworth's 
conclusions nicely anticipate some of Schafer's more well-known points: apprehension is a problem for sound because of urban noise, which he considers to be the main obstacle to sonic design in cities. He argues that high contrast between foreground and background sounds makes sonic spaces more identifiable; and that open space and responsive spaces hold special potencial for experimentation and staging sonic events.

Finally, he points to sonic signs (what Schafer would call soundmarks) as a key to distinctive sonic experiences of the city."

36 “[...] from John Cage’s 4'33” to Pierre Schaeffer's musique concrète to electronic and tape music.”

37 "I use construct rather than concept to note that soundscape theory also has relations to particular cultural practices and to highlight that there is no single, coherent conception of soundscape agreed upon scholars and practitioners." 38 " $[\mathrm{F}]$ or one who believes we are imploding back into aural-tribal age, your knowledge of aural affairs had always seemed too slight."

39 "By aural affairs I am not only referring to music theory, but to the whole development of the acoustic environment - which I call the soundscape."

40 "[S]oundscape is a media concept, and it is a concept that demands its listener experience the broader phenomena of sonic mediations from a stable and surprisingly delicate position."

41 "[T]he edges of his well-centered listening subject are already troubled and shaped by media."

42 "The essence of the soundscape, and indeed the essence of stereo is not physical space or the relation between physical space and its representation. Its essence is a stable audioposition, one from which the entire world is available to be heard."

43 "While soundscape remains a useful term, it raises the question of space, scale and culture without itself giving us the tools to answer it".

\section{Referências}

ARKETTE, Sophie. Sounds like city. Theory, Culture \& Society, Londres, v. 21, n. 1, p. 159- 168, 2004.

HOLZER, Werther. Paisagem, imaginário, identidade: alternativas para o estudo geográfico. In: ROSENDAHL, Zeny et al. (Ed.). Manifestações da Cultura no Espaço. Rio de Janeiro: EdUERJ, 1999. p. 149-168. (Série Geografia Cultural).

INGOLD, Tim. Four objections to the concept of soundscape. In: INGOLD, Tim, Being Alive:Essays on movement, knowledge and description. Londres, Nova York: Routledge, 2011. p. 136-139.

JACOBS, Jane. Morte e vida de grandes cidades. São Paulo: Martins Fontes, 2000.

KELMAN, Ari Y. Rethinking the soundscape. The Senses and Society, Londres, v. 5. n. 2, p. 212234, 2010.

LEFEBVRE, Henri. The production of space. Maiden, Oxford, Victoria: Blackwell Publishing, 1991. $434 \mathrm{p}$.

LYNCH, Kevin. A imagem da cidade. São Paulo: Martins Fontes, 1997. 227 p.

MCLUHAN, Marshall. A Galáxia de Gutenberg: a formação do homem tipográfico. 2. ed. .São Paulo: Editora Nacional, 1977. 390 p.

SCHAFER, R. Murray. A afinação do mundo. São Paulo: Editora UNESP, 2001. p. 381

STERNE, Jonathan. Soundscape, landscape, scape. In: BIJSTERVELD, Karin (Ed.). Soundscapes of urban past: Staged sound as mediated cultural heritage. Bielefeld: Verlag,_2013. p. 181-191. v. 5. (Sound Studies).

STERNE, Jonathan. The stereophonic space of soundscape. In: THÉBERGE, Paul et al. (Ed.). Living Stereo: Histories and cultures of multichannel sound. New York: Continuum, 2015. p. 65-83.

THÉBERGE, Paul; DEVINE, Kyle; EVERRETT, Tom. Introduction: Living Stereo. In: THÉBERGE, Paul et al. (Ed.). Living Stereo: Histories and cultures of multichannel sound. New York: Continuum, 2015. p. 1-34. 
TRUAX, Barry. Acoustic Communication. 2. ed. Westport, Londres: Ablex Publishing, 2001. p. 284.

TRUAX, Barry.Introduction to five village soundscapes (1977). In: SCHAFER, R. Murray (Ed.). Five village soundscapes. 2. ed. Tampere: TAMK University of Applied Sciences, 2009. p. 286289.

Thaís A. Aragão pesquisa som, espaço e mídia. É produtora cultural da Coordenadoria de Comunicação Social e Marketing Institucional da Universidade Federal do Ceará (UFC), onde atua na Rádio Universitária FM. Desenvolveu os estudos "Escuta, gravação, plataforma web: Fazer mapa sonoro como conjunto de práticas de mídia”, tese de doutorado em Comunicação pela UNISINOS, e "Doce som urbano: o triângulo e as territorializações dos vendedores de chegadinho em Fortaleza”, dissertação de mestrado no Programa de Pós-Graduação em Planejamento Urbano e Regional da UFRGS. Atuou como visiting scholar na University of Salford e na Westminster School of Media, Arts and Design (Reino Unido), junto ao Dr. Michael N. Goddard. Também integra o Grupo de Pesquisa Identidade e Território (GPIT-UFRGS). 